\title{
Cytochrome P450 monooxygenase of Acanthamoeba castellanii participates in resistance to polyhexamethylene biguanide treatment
}

\author{
Jian-Ming Huang ${ }^{1}$, Pin-Ju Ko ${ }^{2}$, Chao-Li Huang ${ }^{3}$, Po-Wei Wen ${ }^{4}$, Chun-Hsien Chen ${ }^{5}$, Min-Hsiu Shih ${ }^{6}$, \\ Wei-Chen Lin ${ }^{1,2,5, a, *}$ (D), and Fu-Chin Huang ${ }^{6, a, *}$ \\ ${ }^{1}$ Department of Parasitology, College of Medicine, National Cheng Kung University, Tainan 701, Taiwan \\ 2 Department of Microbiology and Immunology, College of Medicine, National Cheng Kung University, Tainan 701, Taiwan \\ ${ }^{3}$ Institute of Tropical Plant Sciences and Microbiology, National Cheng Kung University, Tainan 701, Taiwan \\ ${ }^{4}$ School of Medicine, College of Medicine, National Cheng Kung University, Tainan 701, Taiwan \\ ${ }^{5}$ Institute of Basic Medical Sciences, College of Medicine, National Cheng Kung University, Tainan 701, Taiwan \\ ${ }^{6}$ Department of Ophthalmology, National Cheng Kung University Hospital, Tainan 701, Taiwan
}

Received 29 June 2021, Accepted 25 October 2021, Published online 10 November 2021

\begin{abstract}
Acanthamoeba spp. are free-living parasites that can cause severe infections such as granulomatous amoebic encephalitis (GAE) and amoebic keratitis (AK). Polyhexamethylene biguanide (PHMB) is a topical application for AK treatment. However, PHMB is not entirely effective against all Acanthamoeba strains or isolates. The mechanisms by which Acanthamoeba protects itself against extreme drug conditions without encystation are still unknown. According to a previous study, cytochrome P450 monooxygenase (CYP450MO) plays an important role in the oxidative biotransformation of numerous drugs related to metabolism. In this study, a CYP450MO fragment was inserted into the pGAPDH-EGFP vector and transfected into Acanthamoeba castellanii. We found that CYP450MO-overexpressing Acanthamoeba had higher survival rates than those of the control cells after PHMB treatment. Moreover, we also found that encystation-related genes such as cellulose synthase I (CSI), encystation-mediating serine proteinase (EMSP), and autophagy-related protein 8 (ATG8) expression levels were not significantly different between Acanthamoeba transfected by pGAPDH-EGFP or pGAPDH-EGFP-CYP450MO. We suggest that Acanthamoeba transfected by pGAPDH-EGFP-CYP450MO may not induce encystation-related genes to resist PHMB treatment. In conclusion, these findings indicate that CYP450MO may be an additional target when PHMB is used for treatment of amoebic keratitis.
\end{abstract}

Key words: Acanthamoeba, Polyhexamethylene biguanide, P450 monooxygenase, Amoebic keratitis.

\begin{abstract}
Résumé - La monooxygénase du cytochrome P450 d'Acanthamoeba castellanii participe à la résistance au traitement par le polyhexaméthylène biguanide. Les Acanthamoeba spp. sont des parasites libres qui peuvent provoquer des infections graves telles que l'encéphalite amibienne granulomateuse (EAG) et la kératite amibienne (KA). Le polyhexaméthylène biguanide (PHMB) est une application topique pour le traitement de la KA. Cependant, le PHMB n'est pas entièrement efficace contre toutes les souches ou isolats d'Acanthamoeba. Les mécanismes par lesquels Acanthamoeba se protège contre des conditions médicamenteuses extrêmes sans enkystation sont encore inconnus. Selon une étude précédente, la monooxygénase du cytochrome P450 (CYP450MO) joue un rôle important dans la biotransformation oxydative de nombreux médicaments liés au métabolisme. Dans cette étude, un fragment CYP450MO a été inséré dans le vecteur pGAPDH-EGFP et transfecté dans Acanthamoeba castellanii. Nous avons constaté que les Acanthamoeba surexprimant le CYP450MO avaient des taux de survie plus élevés que ceux des cellules témoins après un traitement au PHMB. De plus, nous avons également constaté que les gènes liés aux enkystations tels que la cellulose synthase I (CSI), la sérine protéinase médiatrice de l'enkystation (EMSP) et les niveaux d'expression de la protéine 8 liée à l'autophagie (ATG8) n'étaient pas significativement différents entre les Acanthamoeba transfectés par pGAPDH-EGFP ou par pGAPDH-EGFP-CYP450MO. Nous suggérons que les Acanthamoeba transfectés par pGAPDH-EGFP-CYP450MO ne peuvent pas induire les gènes liés à l'enkystation pour résister au traitement PHMB. En conclusion, ces résultats peuvent indiquer que la monooxygénase du cytochrome P450 peut être une cible potentielle pour le traitement par PHMB de la kératite amibienne.
\end{abstract}

*Corresponding authors: wcnikelin@mail.ncku. edu. tw; huangfc@mail.ncku. edu. tw ${ }^{a}$ Wei-Chen Lin and Fu-Chin Huang are contributed equally. 


\section{Introduction}

Acanthamoeba spp. are free-living pathogenic protozoa that are distributed in several environments, including swimming lakes, pools, soil, and dust [6]. Acanthamoeba spp. cause severe sight-threatening infections such as granulomatous amoebic encephalitis (GAE) and amoebic keratitis (AK) [25, 37]. AK has been increasing with contact lens misuse over the past two decades [1, 4, 6, 7]. Acanthamoeba infects patients by causing lid edema, photophobia, epithelial defects, and ring-like stromal infiltrates through injury to the cornea [20,24]. Patients with AK have been treated effectively over the last two decades with topical biguanides; however, current therapy requires surgical intervention because of the failure of medical treatment [15]. Polyhexamethylene biguanide (PHMB) is a polymeric biguanide used as a disinfectant and antiseptic for patients with AK [19, 22]. PHMB is effective against Pseudomonas aeruginosa, Staphylococcus aureus, Escherichia coli, Candida albicans, and Aspergillus brasiliensis [2, 13, 26, 38, 39]. PHMB contains highly charged positive molecules that bind to the phospholipid bilayer of the cell membrane, which is negatively charged, causing penetration, damage, cell lysis, and death of the pathogens [21]. A previous study showed that $0.01 \%$ PHMB could not induce obvious corneal toxicity but lysed Acanthamoeba after treatment in vitro [10, 22]. Combined AK treatment with propamidine, neomycin, and PHMB reduced pain in all patients within 2-4 weeks [36]. PHMB combined with $\mathrm{H}_{2} \mathrm{O}_{2}$ is also used as an ingredient in contact lens-cleaning solutions to prevent corneal infections [30]. Corneal transplantation is another therapeutic approach when topical treatment fails. Nevertheless, corneal transplantation does not eliminate all trophozoites or cysts that can grow in the new cornea. Hence, there are no clinical therapeutic approaches recommended for incorporation into standard practice.

Cytochrome P450 enzymes (CYP450s) involved in drug metabolism are widely identified in different organisms ranging from protozoa to mammals [9, 32, 40]. CYP450s bind and activate two atoms of oxygen from substrates such as peroxide, and lead to hydroxylation [3]. CYP450s also depend on monooxygenase activity, catalyzing the oxidation of endogenous and exogenous substrates, and thereby cause drug degradation [35]. The metabolism of drugs by CYP450s contributes to the formation of products that are less toxic and are excreted easily into cells. Plasmodium berghei and Plasmodium falciparum can induce CYP450s to exhibit resistance to chloroquine treatment [28]. However, clinical isolates of Acanthamoeba with high resistance to PHMB are associated with serious health consequences in Taiwan [10]. Therefore, cytochrome $\mathrm{P} 450$ monooxygenase (CYP450MO) may play an important role in the oxidative biotransformation of numerous drugs during drug metabolism in Acanthamoeba. In this study, we overexpressed CYP450MO in Acanthamoeba to investigate its effects. CYP450MO-overexpressing Acanthamoeba had higher survival rates than those of the control cells after PHMB treatment. We suggest that CYP450MO in Acanthamoeba may catalyze PHMB drug metabolism to enhance survival rates after PHMB treatment. In conclusion, these findings may help to develop potential treatments for AK patients.

\section{Materials and methods}

\section{Acanthamoeba castellanii cultivation}

Trophozoites of A. castellanii (Neff strain, ATCC No. 30010, Pacific Grove, CA, USA) were axenically cultured at $28^{\circ} \mathrm{C}$ in peptone-yeast extract-glucose (PYG) medium $(20 \mathrm{~g} / \mathrm{L}$ proteose peptone, $2 \mathrm{~g} / \mathrm{L}$ yeast extract, $0.1 \mathrm{M}$ glucose, $4 \mathrm{mM}$ $\mathrm{MgSO}_{4}, 3.4 \mathrm{mM}$ sodium citrate, $0.9 \mathrm{mM} \mathrm{Fe}\left(\mathrm{NH}_{4}\right)_{2}\left(\mathrm{SO}_{4}\right)_{2}$, $1.3 \mathrm{mM} \mathrm{Na}_{2} \mathrm{HPO}_{4}$, and $2 \mathrm{mM} \mathrm{K}_{2} \mathrm{HPO}_{4}, \mathrm{pH} 6.5$ ) in cell culture flasks.

\section{Total RNA isolation and cDNA synthesis}

A total RNA Extraction Miniprep System (Viogene, Taiwan) was used to isolate RNA. The total concentration and A260/A280 ratio of mRNA were measured using ND-1000 (NanoDrop, Thermo Fisher Scientific, USA). High-capacity cDNA Reverse Transcription kits (Thermo Fisher Scientific) were used in this study. The reverse transcription conditions were set at the following times and temperatures: $25{ }^{\circ} \mathrm{C}$ for $10 \mathrm{~min}, 37^{\circ} \mathrm{C}$ for $120 \mathrm{~min}$, and $85^{\circ} \mathrm{C}$ for $5 \mathrm{~min}$; finally, the cDNA was kept at $4{ }^{\circ} \mathrm{C}$. The reaction volume was $20 \mu \mathrm{L}$.

\section{Polymerase chain reaction (PCR)}

PCR products were separated on a DNA VIEW (BIOTOOLS Co., Ltd.) - stained gel via agarose gel electrophoresis. The 18S rDNA forward primer F900 was 5' - CCC AGA TCG TTT ACC GTG AA $-3^{\prime}$, and the reverse primer R1100 was $5^{\prime}$ - TAA ATA TTA ATG CCC CCA ACT ATC C - 3', which produced 180-bp amplification bands. CSI forward primer was $5^{\prime}$ - GGC GAA GAA CAC CTG GTT AC - 3', and the reverse primer was $5^{\prime}$ - TGC TCT ACA ACA CGG AGG TG - 3', which produced 239-bp amplification bands. ATG8 forward primer was 5' - AAG GAA GCA CAT GAA GCT GAG C - 3', and the reverse primer was $5^{\prime}-$ CCA TCC TCG TCC TTG TAC TTG $\mathrm{G}-3^{\prime}$, which produced 117-bp amplification bands. EMSP forward primer was $5^{\prime}$ - CAA CTA CAC CCA GGA CAC CC $-3^{\prime}$, and the reverse primer was $5^{\prime}-$ GGT CTA CAA AGC GGG AGA GG - 3' which produced 360-bp amplification bands. All experiments were performed independently in triplicate. Image analysis and quantification were performed using the SmartView Pro 1200 Imager System (Major Science, USA).

\section{Cloning of cytochrome P450 monooxygenase}

Two different protocols were used to clone the CYP450MO using two vectors: the pJET1.2/blunt cloning vector and pGAPDH-EGFP vector [5]. To confirm mRNA sequencing, the amplified CYP450MO was converted to blunt-ended using $\mathrm{Pfu} \mathrm{S}^{+}$DNA polymerase and then ligated with the pJET 1.2/blunt cloning vector. The CYP450MO sequence was amplified by PCR using the ATCC_30010 cellular cDNA as the template. To amplify the cDNA encoding CYP450MO, forward CYP450MO_F (5' - ATG CTG TGG TCG CTG ATT GTT GCG $\left.\mathrm{G}-3^{\prime}\right)$ and reverse CYP450MO_R (5' - GGG 
Table 1. Twenty seven related CYP450 enzymes in Acanthamoeba castellanii.

\begin{tabular}{|c|c|c|}
\hline Name & ID & Description \\
\hline ACA1_290950 & ID: 14926367 & cytochrome p450 superfamily protein [Acanthamoeba castellanii str. Neff] \\
\hline ACA1_175170 & ID: 14925874 & cytochrome p450 superfamily protein [Acanthamoeba castellanii str. Neff] \\
\hline ACA1_174810 & ID: 14925848 & cytochrome p450 superfamily protein [Acanthamoeba castellanii str. Neff] \\
\hline ACA1_254730 & ID: 14923340 & cytochrome p450 superfamily protein [Acanthamoeba castellanii str. Neff] \\
\hline ACA1_046130 & ID: 14922831 & cytochrome p450 superfamily protein [Acanthamoeba castellanii str. Neff] \\
\hline ACA1_385730 & ID: 14922709 & cytochrome p450 superfamily protein [Acanthamoeba castellanii str. Neff] \\
\hline ACA1_183160 & ID: 14922274 & cytochrome p450 superfamily protein [Acanthamoeba castellanii str. Neff] \\
\hline ACA1_278030 & ID: 14921744 & cytochrome p450 superfamily protein [Acanthamoeba castellanii str. Neff] \\
\hline ACA1_277340 & ID: 14921686 & cytochrome P450 monooxygenase, putative [Acanthamoeba castellanii str. Neff] \\
\hline ACA1_054840 & ID: 14921608 & cytochrome p450 superfamily protein $[$ Acanthamoeba castellanii str. Neff] \\
\hline ACA1_236320 & ID: 14919834 & cytochrome p450 superfamily protein [Acanthamoeba castellanii str. Neff] \\
\hline ACA1_372100 & ID: 14918886 & cytochrome p450 superfamily protein [Acanthamoeba castellanii str. Neff] \\
\hline ACA1_065930 & ID: 14918208 & cytochrome p450 superfamily protein [Acanthamoeba castellanii str. Neff] \\
\hline ACA1_202250 & ID: 14916956 & cytochrome p450 superfamily protein [Acanthamoeba castellanii str. Neff] \\
\hline ACA1_178260 & ID: 14916894 & Cytochrome P450, putative [Acanthamoeba castellanii str. Neff] \\
\hline ACA1_019600 & ID: 14915577 & cytochrome p450 superfamily protein [Acanthamoeba castellanii str. Neff] \\
\hline ACA1_241260 & ID: 14913884 & cytochrome p450 superfamily protein [Acanthamoeba castellanii str. Neff] \\
\hline ACA1_100440 & ID: 14913746 & cytochrome p450 superfamily protein [Acanthamoeba castellanii str. Neff] \\
\hline ACA1_095400 & ID: 14913279 & cytochrome p450 superfamily protein [Acanthamoeba castellanii str. Neff] \\
\hline ACA1_375490 & ID: 14912773 & $\begin{array}{l}\text { cytochrome } \mathrm{P} 450 \text {, family } 4 \text {, subfamily b, polypeptide } 1 \text {, putative } \\
\text { [Acanthamoeba castellanii str. Neff] }\end{array}$ \\
\hline ACA1_033760 & ID: 14912706 & cytochrome p450 superfamily protein [Acanthamoeba castellanii str. Neff] \\
\hline ACA1_353190 & ID: 14912519 & cytochrome p450 superfamily protein [Acanthamoeba castellanii str. Neff] \\
\hline ACA1_338060 & ID: 14911366 & cytochrome p450 superfamily protein [Acanthamoeba castellanii str. Neff] \\
\hline ACA1_096520 & ID: 14913466 & cytochrome p450 superfamily protein [Acanthamoeba castellanii str. Neff] \\
\hline ACA1_096500 & ID: 14913225 & cytochrome p450 superfamily protein [Acanthamoeba castellanii str. Neff] \\
\hline ACA1_374460 & ID: 14912855 & cytochrome p450 superfamily protein [Acanthamoeba castellanii str. Neff] \\
\hline ACA1_139550 & ID: 14914785 & nadph cytochrome P450, putative [Acanthamoeba castellanii str. Neff] \\
\hline
\end{tabular}

CAG TGG TAC GTT TGC GGC AAA - 3') primers were used. The CYP450MO was cloned into the pJET1.2/blunt cloning vector using a CloneJET PCR Cloning kit (Thermo Fisher Scientific). A CYP450MO fragment was inserted into the pGAPDH-EGFP vector using NdeI/SpeI sites. To amplify the cDNA encoding CYP450MO, forward NdeI_CYP450MO_F $\left(5^{\prime}\right.$ - AAC ATA TGC TGT GGT CGC TGA TTG TTG CGG - $\left.3^{\prime}\right)$ and reverse SpeI_CYP450MO_R (5' - ACA CTA GTG GGC AGT GGT ACG TTT GCG - $3^{\prime}$ ) primers were used. All plasmids were transformed to DH5 $\alpha$ competent E. coli for replication and construction.

\section{Phylogenetic analysis of AcCYP450MO}

We conducted blastp with the peptide sequence of AcCYP450MO against the NCBI nr database (National Center for Biotechnology Information) and retrieved the sequences of the top 100 hits. These sequences were aligned with the "hmmalign" program of the HMMER package v.3.1b2, according to the "cytochrome P450" domain in the pfam database. With the best protein substitution model "JTT + G + I" predicted by MEGA v.7.0 [17], as well as a bootstrap analysis of 100, a maximum likelihood phylogeny was reconstructed with raxml v.8.2.12 [33]. In addition, the functional domain of cytochrome $\mathrm{P} 450$ was predicted with the "hmmscan" program of the HMMER package. Structural similarity was assessed by an online tool "Phyre2" [14].

\section{Cell electroporation of $\boldsymbol{A}$. castellanii}

For electroporation, cells were counted using a hemocytometer and centrifuged at $3000 \mathrm{rpm}$ for $3 \mathrm{~min}$ to remove the medium. Acanthamoeba cells were resuspended in PAS to a final count of $5 \times 10^{6}$ cells $/ \mathrm{mL}$ and placed in an Eppendorf tube. Ten micrograms of plasmid DNA were added to the Eppendorf tube, followed by PAS to a final volume of $800 \mu \mathrm{L}$. The mixture was gently mixed and dispensed into a 4-mm cuvette. Using Gene Pulser Xcell ${ }^{\mathrm{TM}}$, the protocol was set as follows: $150 \mathrm{~V}, 10 \mathrm{~ms}$. After electroporation, the cuvettes containing cells were placed on ice for $10 \mathrm{~min}$, and cells were transferred to a T-75 flask containing PYG for incubation at $28{ }^{\circ} \mathrm{C}$ overnight. Stable transformants were selected using $40 \mu \mathrm{g} / \mathrm{mL}$ Geneticin (G418).

\section{Survival rates of CYP450MO-overexpressing A. castellanii}

CYP450MO-overexpressing amoeba cells were seeded at a density of $5 \times 10^{6}$ cells $/ \mathrm{mL}$ in a 6 -well plate and treated with $0.01 \%$ PHMB for different times, counted using a hemocytometer, and stained using trypan blue.

\section{Statistical analysis}

Data are presented as mean \pm standard deviation (SD) from three independent experiments. Student's $t$-test was used 


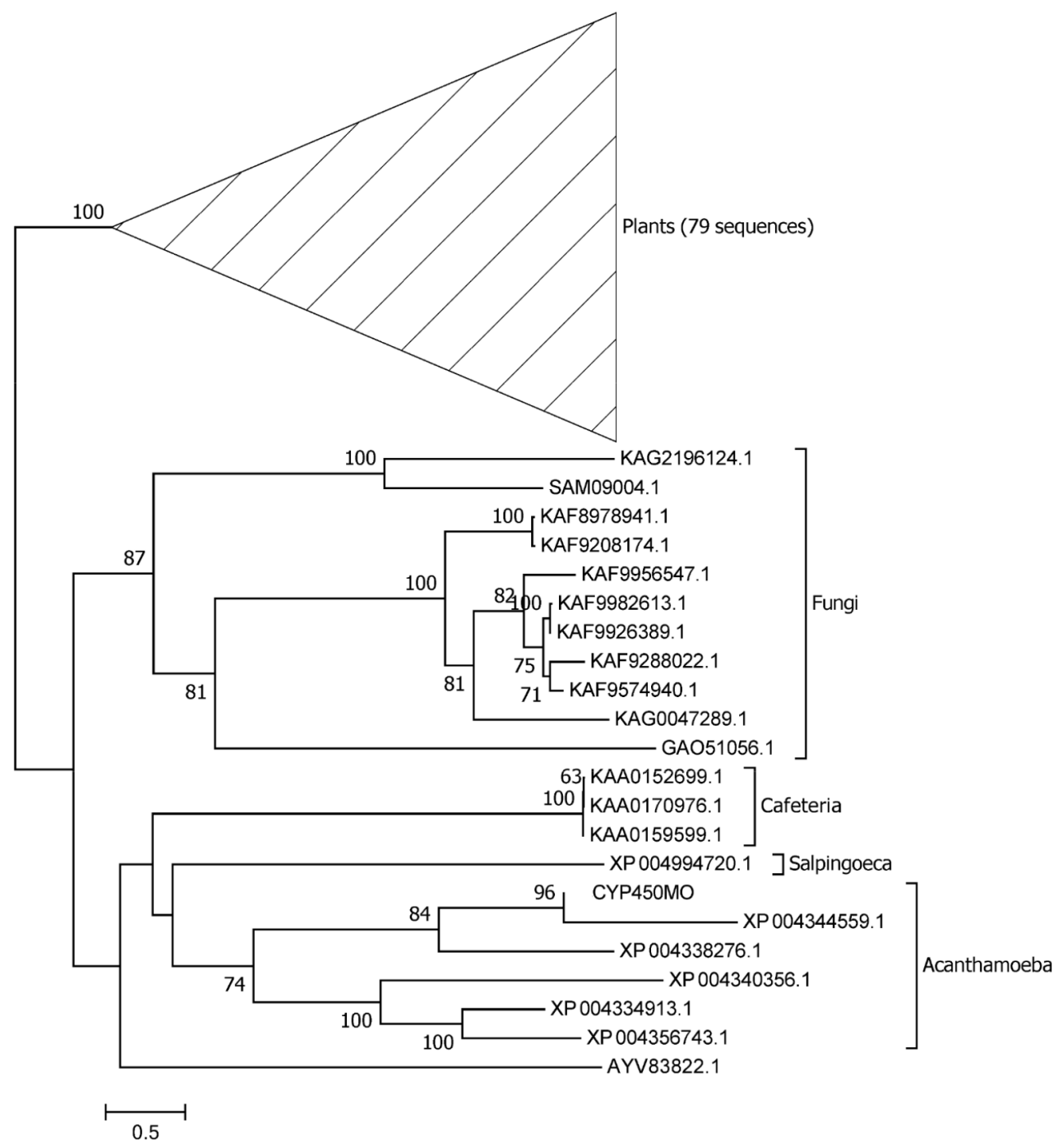

Figure 1. Maximum-likelihood phylogeny of the top 100 peptides closely related to CYP450MO. The numbers next to branches indicate bootstrap support.

for statistical analysis. Statistical significance was set at $p<0.05$.

\section{Results}

\section{The sequencing of cytochrome P450 monooxygenase}

CYP450s are widely distributed throughout different organisms ranging from protozoa to mammals [9, 32, 40]. In Acanthamoeba, we found 27 CYP450 enzymes (Table 1); moreover, only one CYP450 contained a monooxygenase domain (cytochrome P450 monooxygenase, ACA1_277340) to catalyze a variety of substrates with one oxygen atom [35]. To confirm the mRNA sequence of CYP450MO, we amplified the cDNA using ATCC_30010 cellular cDNA as the template. Compared to the sequences in the NCBI-nr database, we found many differences in the CYP450MO of ATCC_30010 cellular cDNA. We conducted a phylogenetic analysis on CYP450MO and the most similar peptides in GenBank. All peptides of Acanthamoeba formed a monophyletic clade, next to sequences of Salpingoeca (a Choanoflagellate) (Fig. 1). In the clade, CYP450MO was closely related to ACA1_277340 (XP004344559.1). When comparing with the coding sequence with ACA1_277340, their 5' and $3^{\prime}$ ends were identical, while the major difference occurred in the completeness of the cytochrome P450 domain (Fig. 2). CYP450MO possessed a full structure, but the domain was truncated in ACA1_277340 (Fig. 2B). Moreover, phyre2 analysis indicated that CYP450MO showed $99.9 \%$ confidence on a high similarity to the structure of human cytochrome P450 2a6. These results indicated that CYP450MO was more likely to show full function than that of ACA1_277340.

\section{The function of CYP450MO in Acanthamoeba}

To determine whether CYP450MO of Acanthamoeba can affect PHMB drug degradation, the enzyme was overexpressed 
A

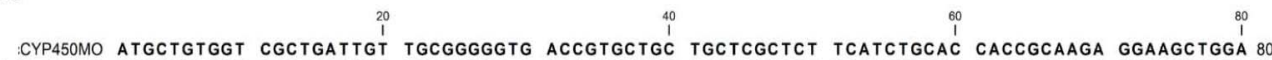
ACA1_277340 ATGCTGTGGT CGCTGATTGT TGCGGGGGT ACCGTGCTGC TGCTCGCTCT TCATCTGCAC CACCGCAAGA GGAAGCTGGA 80 Conservation 100 ${ }^{120}$ 140
1

CYP450MO CATACCTGGT CCTTACCAGC TGCCCATCTT GTGCAACCTC ATCGACACTT TCCGCCACAA GCACCGCCTC CAAGACTACA 160 ACA1_277340 CATACCTGGT GCTTACCAGC TGCCCATCTT GTGCAACCTC ATCGACACTT TCCGCCACAA GCACCGCCTC CAAGACTACA 160 Conservation

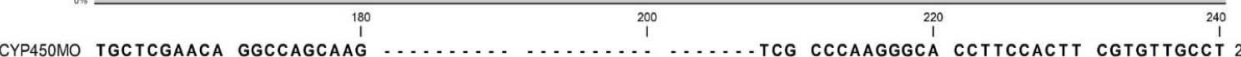

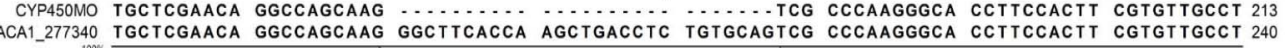
Conservation 260 ${ }^{280}$ 300

CYP450MO ITCGGGGGCG ATACGGACGT GATGGTCACA GACCCGCCCT CGGTCGAATA CATCGTCAAG ACCAACTTCG AAAACTTCAC 203 ACA1_277340 TTCGGGGGCG ATACGGACGT GATGGTCACA GACCCGCCCT GGGTCGAATA CATCCTCAAG ACCAACTTCG AAAACTTCAC 320 Conservation

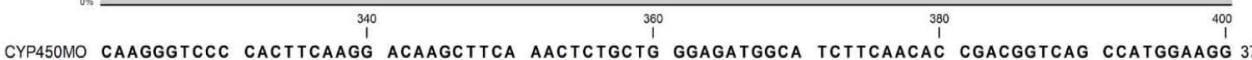

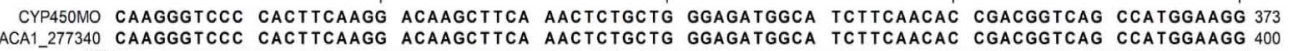
Conservation 420 440 460

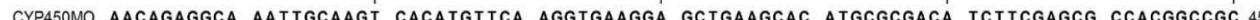
Conservation

$\begin{array}{llcl}500 & 520 & 540 & 560 \\ 1\end{array}$ CYP450MO GAGCTGCTGC AAATCCTCGA CAAGGCGGCC GACACCGGCG TGCCCATCGA TGTGGCCGAT CTCTTCTTCA GGTACACCCT 533

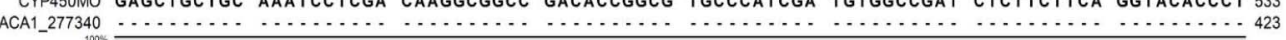
Conservation 580 600
1 $\stackrel{620}{1}$

CYP450MO GGACTCGATC GGAGAGATCG CTTTTGGCTT CAACGTCGAC TCCATGCACA AGGACTGCTC CITCTCGCAA GCCTTCGACG O13 ACA1_277340 .........ATC GGAGAGATCG CTTTTTGGCTT CAACGTCGAC TCCATGCACA AGGACTGCTC CTTCTCGCAA GCCTTCGACG 496 Conservation

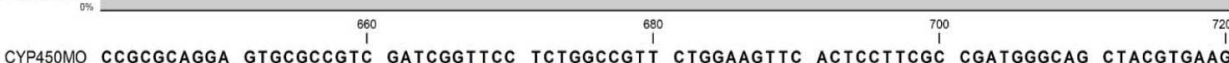
CYP450MO CCGCGCAGGA GTGCGCCGTC GATCGGTTCC TCTGGCCGTT CTGGAAGTTC ACTCCTTCGC CGATGGGCAG CTACGTGAAG 693
ACA1_277340 CCGCGCAGGA GTGCGCCGTC GATCGGTTCC TCTGGCCGTT CTGGAAGTTC ACTCCTTCGC CGATGGCCAG CTACGTGAA 576 Conservation

740
1

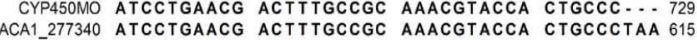
1005
Conservation B

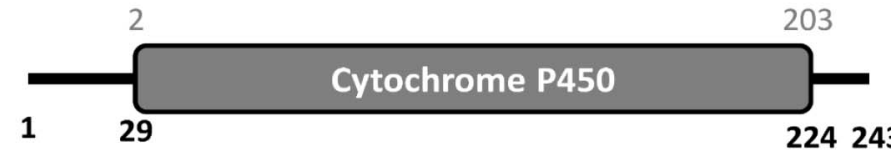

\section{CYP450MO}

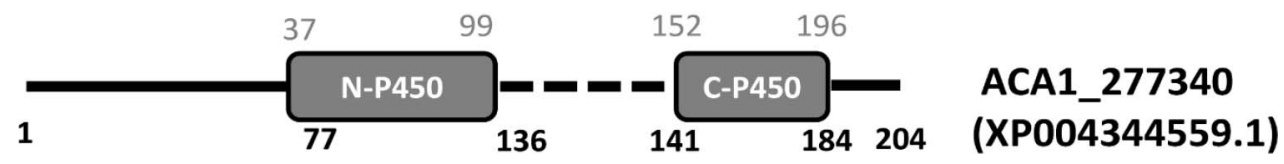

Figure 2. Sequence alignment between CYP450MO and ACA1_277340. (A) Alignment of coding sequences. (B) Schematic representation of the alignment of the cytochrome $\mathrm{P} 450$ domain. The numbers in black indicate the position on peptides, while the numbers in grey stand for the position of the hmm model of cytochrome p450 in the pfam annotation database.

by the pGAPDH-EGFP vector. A CYP450MO fragment was inserted into the pGAPDH-EGFP vector using NdeI/SpeI sites (Fig. 3A). After transfection in Acanthamoeba by electroporation for 14 days, the pGAPDH-EGFP-CYP450MO vector was expressed. To confirm that the pGAPDH-EGFPCYP450MO vector was transfected into Acanthamoeba, the DNA extracted from Acanthamoeba was amplified using the pGAPDH-EGFP primers (Fig. 3B). The EGFP-CYP450MO fusion protein was also expressed in Acanthamoeba using a $\mathrm{Cell}^{\mathrm{R}}$ microscope (Olympus America, Inc., USA) for 7 days (Fig. 3C).
Acanthamoeba-transfected pGAPDH-EGFP-CYP450MO vectors were treated with $0.01 \%$ PHMB. The results showed that the survival rates of Acanthamoeba-transfected pGAPDH-EGFP-CYP450MO vector were higher than those of the control at 1, 16, and $24 \mathrm{~h}$ (Fig. 4). Hence, we suggest that Acanthamoeba overexpressing CYP450MO may be resistant to PHMB drug, enhancing survival rates.

\section{CYP450MO and encystation in Acanthamoeba}

A previous study showed that clinical isolates can resist drugs by encystation to avoid environmental stress [10]. 
A

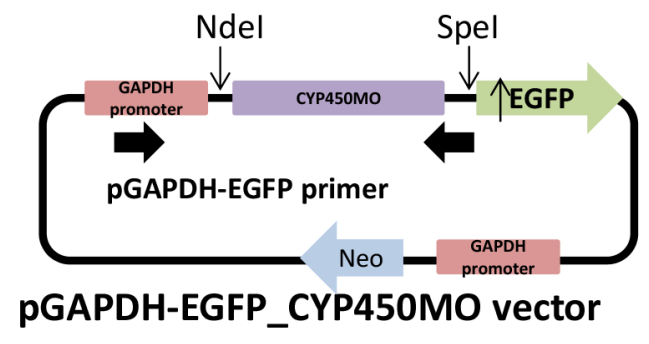

B

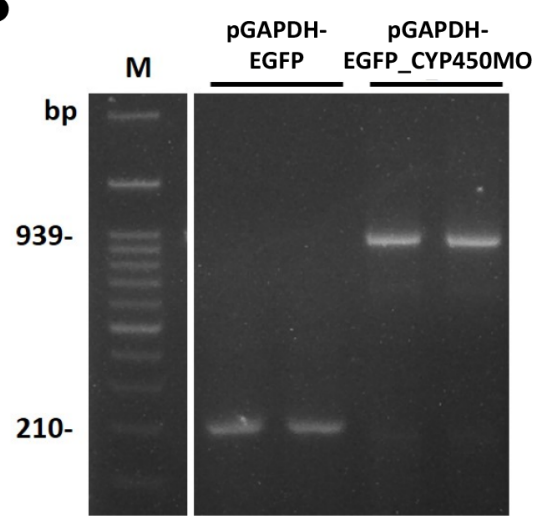

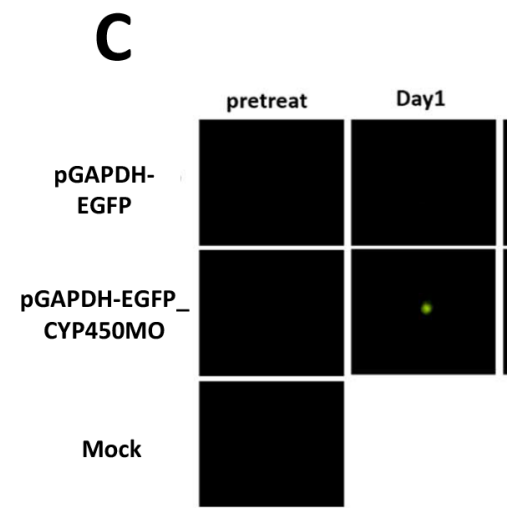
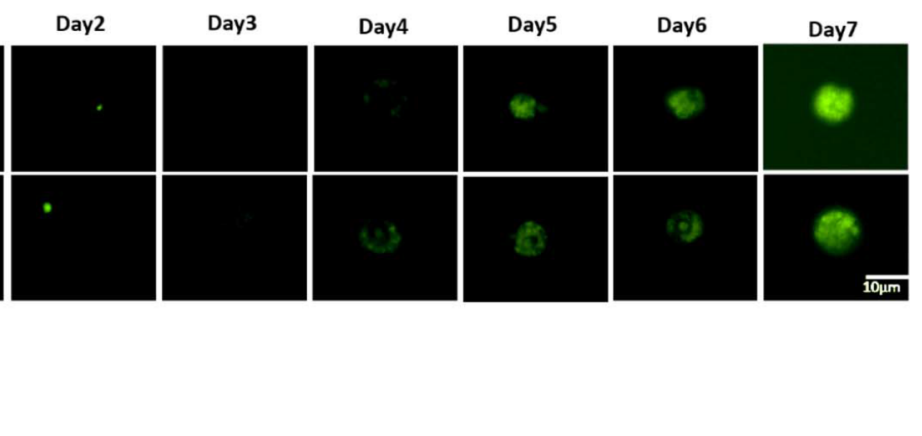

Figure 3. CYP450MO overexpression in Acanthamoeba (ATCC_30010). (A) Schematic of the pGAPDH-EGFP-CYP450MO vector. (B) Genomic DNA of Acanthamoeba transfected in the pGAPDH-EGFP-CYP450MO vector detected by PCR. (C) Acanthamoeba transfected with pGAPDH-EGFP and pGAPDH-EGFP-CYP450MO vector (green) incubated for 7 days and examined using a fluorescence microscope.

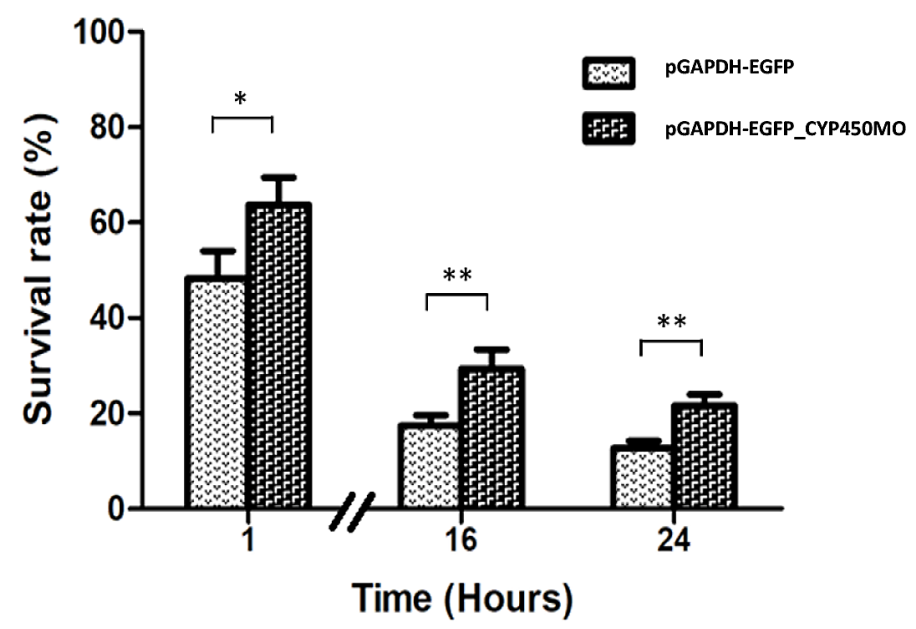

Figure 4. Survival rate of Acanthamoeba treated with PHMB. Survival rate of Acanthamoeba cells transfected with pGAPDH-EGFP and pGAPDH-EGFP-CYP450MO vector incubated with $0.01 \%$ PHMB for 1,16 , and $24 \mathrm{~h}$. Data are presented as mean \pm standard deviation (SD).

To determine whether Acanthamoeba-transfected pGAPDHEGFP-CYP450MO vector induced encystations to avoid PHMB drug lysis, gene-related encystations were detected. CSI, EMSP and ATG8 identified in Acanthamoeba are involved in the encystation mechanism $[16,27]$. The results showed that
ATG8 expression was not significantly different between Acanthamoeba-transfected pGAPDH-EGFP and pGAPDHEGFP-CYP450MO (Fig. 5A). CSI and EMSP expression levels were also not significantly different between Acanthamoebatransfected pGAPDH-EGFP and pGAPDH-EGFP-CYP450MO 
A
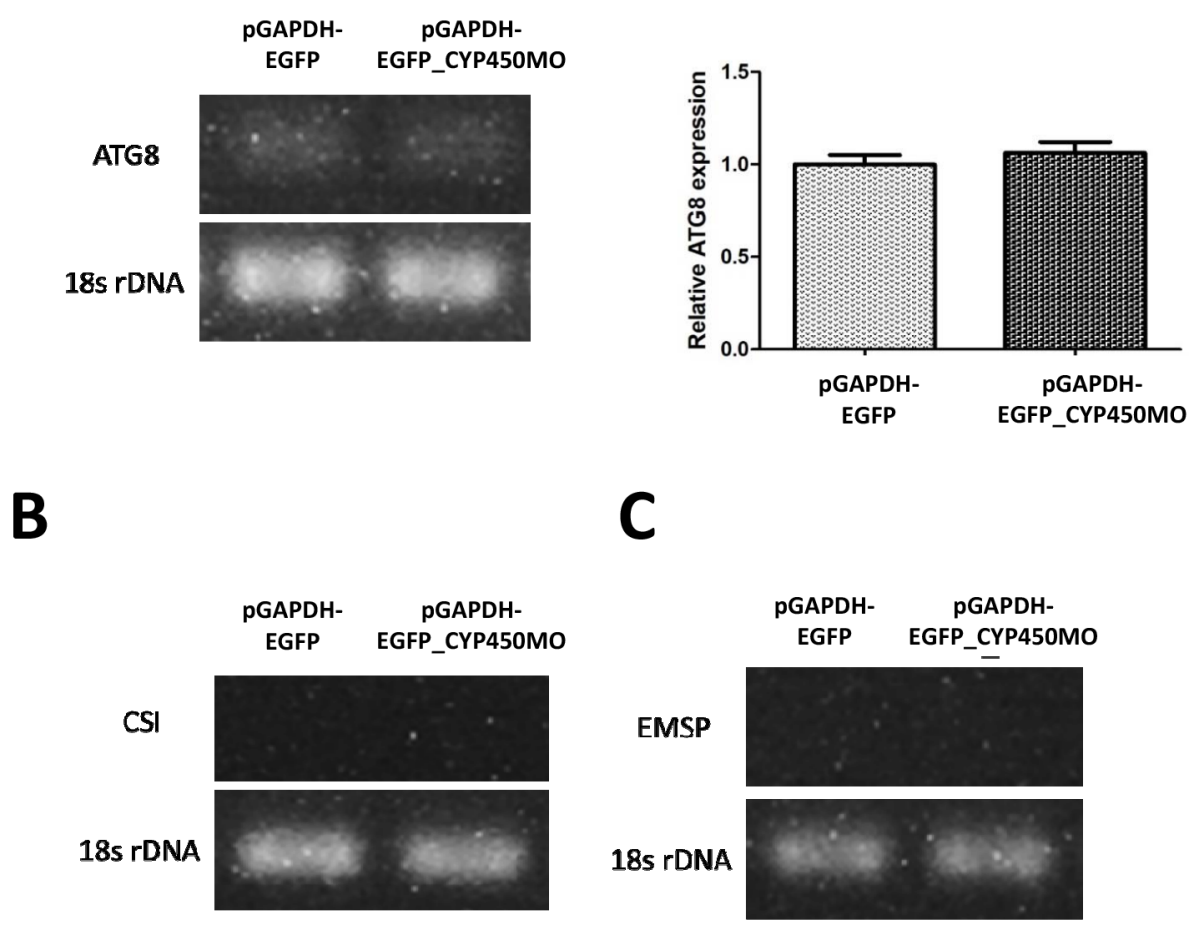

Figure 5. mRNA expression of encystation genes in Acanthamoeba transfected with pGAPDH-EGFP and pGAPDH-EGFP-CYP450MO vector. mRNA expression of ATG8 (A), CSI (B), and EMSP (C). 18s rDNA expression was used as the control $\left({ }^{*} p \leq 0.05\right)$.

(Figs. 5B and 5C). Hence, we suggest that Acanthamoebatransfected pGAPDH-EGFP-CYP450MO may not induce encystation to resist PHMB drug lysis.

\section{Discussion}

Acanthamoeba castellanii has 27 CYP450 genes compared to the 57 CYP450 genes in the human genome [29]. The CYP450 genes related to drug metabolism in humans are CYP2C9, CYP2C19, CYP2D6, and CYP3A4 [11]. In nematodes, Caenorhabditis elegans encodes 80 CYP450 genes. Some CYPs in C. elegans such as cyp35a2, cyp35a5, and cyp35c1 play a role in albendazole (ABZ), an anti-helminthic medication $[8,18]$. However, in protozoa such as Toxoplasma gondii, the CYP450 gene exists as a single copy. The CYP450 of T. gondii plays an important role in developing resistance to drugs such as quinine, mefloquine, and clarithromycin [40]. In this study, we found 27 related CYP450 enzymes in A. castellanii (Table 1). A previous study showed that CYP450 genes in humans were observed to enhance gene diversity by alternative RNA splicing [34]. Therefore, it is likely that CYP450s are produced from the Acanthamoeba gene by alternative splicing to metabolize different drugs.

In this study, CYP450MO induced PHMB drug metabolism for the survival of Acanthamoeba, as CYP450MO overexpression enhanced the resistance of Acanthamoeba. Moreover, in previous studies, strains resistant to encystation were also transformed into pseudocysts or cysts under the effects of PHMB drug stress [10, 23]. ATG8 in Acanthamoeba encystation plays an important role in autophagy against drug therapy [12]. CSI and EMSP have also been identified in Acanthamoeba and are involved in the encystation mechanism [16, 27]. However, ATG8, CSI, and EMSP levels were not significantly different between Acanthamoeba-transfected pGAPDH-EGFP and pGAPDH-EGFP-CYP450MO (Fig. 5). Hence, we suggest that Acanthamoeba may not express encystation-related genes against PHMB drug lysis.

CYP450s are known to catalyze a variety of chemical reactions and attack substrates from electron transfer chains. On the electron transfer chains, CYP450s incorporate oxygen atoms into the substrate molecule by transferring electrons from $\mathrm{NAD}(\mathrm{P}) \mathrm{H}$ [31]. Monooxygenase systems depend on monooxygenase activity catalyzing one oxygen atom in the substrate molecule. Many drug metabolic processes catalyzed by monooxygenase involve the oxidation of endogenous and exogenous substrates [35]. In this study, we also found that the survival rates of Acanthamoeba-transfected pGAPDHEGFP-CYP450MO vector were higher than those of the control after PHMB treatment (Fig. 4). Hence, we suggest that CYP450MO in Acanthamoeba may catalyze PHMB drug metabolism to exogenous substrates and be secreted into the extracellular environment. In the future, we aim to focus on CYP450MO as a drug target to potentially treat AK.

\section{Conclusions}

In this study, we overexpressed CYP450MO in Acanthamoeba to investigate PHMB drug resistance. Acanthamoeba 
with CYP450MO-overexpression had higher survival rates than those of the control cells after PHMB treatment. We suggest that CYP450MO in Acanthamoeba may catalyze PHMB drug metabolism to enhance survival rates after PHMB treatment.

\section{Availability of data and materials}

Data supporting the conclusions of this article are included within the article. The datasets used and/or analyzed during the present study are available from the corresponding author upon reasonable request.

\section{Competing interests}

All authors declare that they have no conflicts of interest.

\section{Funding}

This research was supported by the Ministry of Science and Technology (MOST) through funding to WCL (grant MOST 110-2628-B-006-32) and by the National Cheng Kung University Hospital through funding to $\mathrm{FCH}$.

Acknowledgements. We acknowledge Professor Yeonchul Hong (Department of Parasitology, Kyungpook National University School of Medicine) for providing pGAPDH-EGFP plasmid and Professor Jyh-Wei Shin (Department of Parasitology, College of Medicine, National Cheng Kung University) for providing reagents, materials, and analysis tools. We would like to thank Editage (https://www.editage.com) for English language editing.

\section{References}

1. Allen MJ, Morby AP, White GF. 2004. Cooperativity in the binding of the cationic biocide polyhexamethylene biguanide to nucleic acids. Biochemical and Biophysical Research Communications, 318(2), 397-404.

2. Allen MJ, White GF, Morby AP. 2006. The response of Escherichia coli to exposure to the biocide polyhexamethylene biguanide. Microbiology, 152(4), 989-1000.

3. Anzenbacher P, Anzenbacherova E. 2001. Cytochromes P450 and metabolism of xenobiotics. Cellular and Molecular Life Sciences, 58(5-6), 737-747.

4. Auran JD, Starr MB, Jakobiec FA. 1987. Acanthamoeba keratitis. Cornea, 6(1), 2-26.

5. Bateman E. 2010. Expression plasmids and production of EGFP in stably transfected Acanthamoeba. Protein Expression and Purification, 70(1), 95-100.

6. Brown T, Cursons R, Keys E. 1982. Amoebae from Antarctic soil and water. Applied and Environmental Microbiology, 44c (2), 491-493.

7. Broxton P, Woodcock P, Gilbert P. 1983. A study of the antibacterial activity of some polyhexamethylene biguanides towards Escherichia coli ATCC 8739. Journal of Applied Bacteriology, 54(3), 345-353.

8. Cvilink V, Skalova L, Szotakova B, Lamka J, Kostiainen R, Ketola RA. 2008. LC-MS-MS identification of albendazole and flubendazole metabolites formed ex vivo by Haemonchus contortus. Analytical and Bioanalytical Chemistry, 391(1), 337-343.
9. Guengerich FP. 2008. Cytochrome p450 and chemical toxicology. Chemical Research in Toxicology, 21(1), 70-83.

10. Huang F-C, Shih M-H, Chang K-F, Huang J-M, Shin J-W, Lin W-C. 2017. Characterizing clinical isolates of Acanthamoeba castellanii with high resistance to polyhexamethylene biguanide in Taiwan. Journal of Microbiology, Immunology and Infection, 50(5), 570-577.

11. Ingelman-Sundberg M. 2004. Human drug metabolising cytochrome P450 enzymes: properties and polymorphisms. Naunyn-Schmiedeberg's Archives of Pharmacology, 369(1), 89-104.

12. Jha BK, Jung H-J, Seo I, Kim HA, Suh S-I, Suh M-H, Baek WK. 2014. Chloroquine has a cytotoxic effect on Acanthamoeba encystation through modulation of autophagy. Antimicrobial Agents and Chemotherapy, 58(10), 6235-6241.

13. Kamaruzzaman NF, Chong SQ, Edmondson-Brown KM, NtowBoahene W, Bardiau M, Good L. 2017. Bactericidal and antibiofilm effects of polyhexamethylene Biguanide in models of intracellular and biofilm of Staphylococcus aureus isolated from bovine mastitis. Frontiers in Microbiology, 8, 1518.

14. Kelley LA, Mezulis S, Yates CM, Wass MN, Sternberg MJ. 2015. The Phyre2 web portal for protein modeling, prediction and analysis. Nature Protocols, 10(6), 845-858.

15. Kitzmann AS, Goins KM, Sutphin JE, Wagoner MD. 2009. Keratoplasty for treatment of Acanthamoeba keratitis. Ophthalmology, 116(5), 864-869.

16. Kliescikova J, Kulda J, Nohynkova E. 2011. Stress-induced pseudocyst formation-a newly identified mechanism of protection against organic solvents in Acanthamoebae of the T4 genotype. Protist, 162(1), 58-69.

17. Kumar S, Stecher G, Tamura K. 2016. MEGA7: molecular evolutionary genetics analysis version 7.0 for bigger datasets. Molecular Biology and Evolution, 33(7), 1870-1874.

18. Laing ST, Ivens A, Laing R, Ravikumar S, Butler V, Woods DJ, Gilleard JS. 2010. Characterization of the xenobiotic response of Caenorhabditis elegans to the anthelmintic drug albendazole and the identification of novel drug glucoside metabolites. Biochemical Journal, 432(3), 505-516.

19. Larkin D, Kilvington S, Dart J. 1992. Treatment of Acanthamoeba keratitis with polyhexamethylene biguanide. Ophthalmology, 99(2), 185-191.

20. Li M-L, Shih M-H, Huang F-C, Tseng S-H, Chen C-C. 2012. Treatment of early Acanthamoeba keratitis with alcohol-assisted epithelial debridement. Cornea, 31(4), 442-446.

21. Lim N, Goh D, Bunce C, Xing W, Fraenkel G, Poole TR, Ficker L. 2008. Comparison of polyhexamethylene biguanide and chlorhexidine as monotherapy agents in the treatment of Acanthamoeba keratitis. American Journal of Ophthalmology, 145(1), 130-135.

22. Lim C-C, Peng I-C, Huang Y-H. 2020. Safety of intrastromal injection of polyhexamethylene biguanide and propamidine isethionate in a rabbit model. Journal of Advanced Research, 22, $1-6$.

23. Lloyd D. 2014. Encystment in Acanthamoeba castellanii: a review. Experimental Parasitology, 145, S20-S27.

24. Lorenzo-Morales J, Khan NA, Walochnik J. 2015. An update on Acanthamoeba keratitis: diagnosis, pathogenesis and treatment. Parasite, 22, 10.

25. McCulley JP, Alizadeh H, Niederkorn JY. 1995. Acanthamoeba keratitis. Eye \& Contact Lens, 21(1), 73-76.

26. Mikić IM, Cigić L, Kero D, Govorko DK, Mehičić GP, Simeon P. 2018. Antimicrobial effectiveness of polyhexamethylene biguanide on Enterococcus faecalis, Staphylococcus epidermidis and Candida albicans. Medicinski Glasnik, 15(2), 132-138. 
27. Moon E-K, Hong Y, Chung D-I, Kong H-H. 2012. Cysteine protease involving in autophagosomal degradation of mitochondria during encystation of Acanthamoeba. Molecular and Biochemical Parasitology, 185(2), 121-126.

28. Ndifor AM, Ward SA, Howells RE. 1990. Cytochrome P-450 activity in malarial parasites and its possible relationship to chloroquine resistance. Molecular and Biochemical Parasitology, 41(2), 251-257.

29. Nebert DW, Wikvall K, Miller WL. 2013. Human cytochromes P450 in health and disease. Philosophical Transactions of the Royal Society B: Biological Sciences, 368(1612), 20120431.

30. Pinna A. 2002. Acanthamoeba and disinfecting contact lens solutions. British Journal of Ophthalmology, 86(12), 1461-1462.

31. Porter T, Coon M. 1991. Cytochrome P-450. Multiplicity of isoforms, substrates, and catalytic and regulatory mechanisms. Journal of Biological Chemistry, 266(21), 13469-13472.

32. Seliskar M, Rozman D. 2007. Mammalian cytochromes P450 importance of tissue specificity. Biochimica et Biophysica Acta (BBA) - General Subjects, 1770(3), 458-466.

33. Stamatakis A. 2014. RAxML version 8: a tool for phylogenetic analysis and post-analysis of large phylogenies. Bioinformatics, 30(9), 1312-1313.

34. Turman CM, Hatley JM, Ryder DJ, Ravindranath V, Strobel HW. 2006. Alternative splicing within the human cytochrome P450 superfamily with an emphasis on the brain: the convolution continues. Expert Opinion on Drug Metabolism \& Toxicology, 2(3), 399-418.

35. Urlacher VB, Girhard M. 2012. Cytochrome P450 monooxygenases: an update on perspectives for synthetic application. Trends in Biotechnology, 30(1), 26-36.

36. Varga JH, Wolf TC, Jensen HG, Parmley VC, Rowsey JJ. 1993. Combined treatment of Acanthamoeba keratitis with propamidine, neomycin, and polyhexamethylene biguanide. American Journal of Ophthalmology, 115(4), 466-470.

37. Visvesvara GS, Moura H, Schuster FL. 2007. Pathogenic and opportunistic free-living amoebae: Acanthamoeba spp., Balamuthia mandrillaris, Naegleria fowleri, and Sappinia diploidea. FEMS Immunology \& Medical Microbiology, 50(1), 1-26.

38. Walls G, Noonan L, Wilson E, Holland D, Briggs S. 2013. Successful use of locally applied polyhexamethylene biguanide as an adjunct to the treatment of fungal osteomyelitis. Canadian Journal of Infectious Diseases and Medical Microbiology, 24(2), 109-112.

39. Werthen M, Davoudi M, Sonesson A, Nitsche D, Mörgelin M, Blom K, Schmidtchen A. 2004. Pseudomonas aeruginosa-induced infection and degradation of human wound fluid and skin proteins ex vivo are eradicated by a synthetic cationic polymer. Journal of Antimicrobial Chemotherapy, 54(4), 772-779.

40. Zhang X, Zhang T, Liu J, Li M, Fu Y, Xu J, Liu Q. 2017. Functional characterization of a unique cytochrome $\mathrm{P} 450$ in Toxoplasma gondii. Oncotarget, 8(70), 115079.

Cite this article as: Huang J.-M, Ko P.-J, Huang C.-L, Wen P.-W, Chen C.-H, Shih M.-H, Lin W.-C \& Huang F.-C. 2021. Cytochrome P450 monooxygenase of Acanthamoeba castellanii participates in resistance to polyhexamethylene biguanide treatment. Parasite $28,77$.

\section{PARASTE}

An international open-access, peer-reviewed, online journal publishing high quality papers on all aspects of human and animal parasitology

Reviews, articles and short notes may be submitted. Fields include, but are not limited to: general, medical and veterinary parasitology; morphology, including ultrastructure; parasite systematics, including entomology, acarology, helminthology and protistology, and molecular analyses; molecular biology and biochemistry; immunology of parasitic diseases; host-parasite relationships; ecology and life history of parasites; epidemiology; therapeutics; new diagnostic tools.

All papers in Parasite are published in English. Manuscripts should have a broad interest and must not have been published or submitted elsewhere. No limit is imposed on the length of manuscripts.

Parasite (open-access) continues Parasite (print and online editions, 1994-2012) and Annales de Parasitologie Humaine et Comparée (1923-1993) and is the official journal of the Société Française de Parasitologie. 